\title{
Fundus changes in incontinentia pigmenti (Bloch-Sulzberger syndrome): a case report
}

\author{
R. B. JAIN AND G. S. WILLETTS \\ From the Department of Ophthalmology, York District Hospital
}

SUMMARY A case of incontinentia pigmenti is reported with fundus changes in 1 eye. She had microaneurysms temporal to the macula, with an abnormal branch of inferior temporal vein. There was extensive retinitis proliferans in the upper temporal equatorial region, which showed leakage on fluorescein angiography.

The ocular and systemic changes in incontinentia pigmenti are reviewed here and a case is reported with fundus changes which have not been described previously.

Incontinentia pigmenti (IP) is characterised by ectodermal defects, with occasional associated mesodermal abnormalities. The typical skin lesions are blue-grey to chocolate-brown arranged along the naevus lines of Blaschko. These occur on the torso and the extremities. The disease can be differentiated into 2 clinical types: Bloch-Sulzberger and Naegeli. The Bloch-Sulzberger syndrome occurs predominantly in females and is usually present at birth. There is patchy, splash-like cutaneous pigmentation with whorls and splotches of pigment. It is associated with frequent ocular changes, absence or malformations of the teeth, and alopecia. The Naegeli type occurs in both males and females and appears after 2 years of age. There is a reticular type of pigmentation, sometimes associated with hypohidrosis, unhealthy and spotted teeth, and keratosis of palms and soles.

The ocular anomalies occur in about a quarter of cases of Bloch-Sulzberger syndrome. They include cataract, uveitis, blue sclerae, conjunctival pigmentation, strabismus, nystagmus, papillitis, optic atrophy, metastatic ophthalmitis, pseudoglioma, retrolental fibroplasia, phthisis bulbi, and microphthalmos.

\section{Case report}

The patient was a female born on 14 April 1962 at full term by normal delivery with a birth weight of

Address for reprints: G. S. Willetts, Department of Ophthalmology, York District Hospital, Wigginton Road, York YO3 $7 \mathrm{HE}$
$6 \mathrm{lb} 12 \mathrm{oz}(3060 \mathrm{~g})$. The pregnancy was uneventful and the mother did not receive any medication during pregnancy. The patient has 4 siblings and they are all healthy. At birth she was noticed to have reddened skin and blisters on legs and arms, and when 3 days old she was admitted to hospital with erythema and bullous eruptions involving scalp, ears, hard palate, forearms, buttocks, thighs, knees, and calves. By 9 days of age the lesions had extended to the chest, and the patient became very toxic. The general condition started improving in the next 3 weeks with nursing care. She was given several courses of antibiotics, as she was very susceptible to upper respiratory infections. The condition stabilised by 3 months of age, and the lesions started becoming pigmented (Fig. 1). It was clinically diagnosed as epidermolysis bullosa, but the diagnosis was changed to IP as pigmentation started. At the age of 4 months skin biopsy established the diagnosis of IP.

At present the patient has classical bizarre whorls and streaks of pigmentation on the trunk and the left groin (Fig. 2). She is thinly built, with diffuse alopecia and very sparse hairs on the scalp. She has marked dental anomalies, having only a few pegged teeth.

OCULAR HISTORY

In October 1973 she was found to have defective visual acuity in the right eye during a routine school medical examination. Her visual acuity was $6 / 9$ in the right eye and $6 / 5$ in the left eye. The right vision improved to $6 / 5$ with a small myopic correction. In April 1976 fundus changes were noticed in the right eye, and she was referred to the eye department of York District Hospital. She had no ocular symptoms.

On examination she was orthophoric and the 


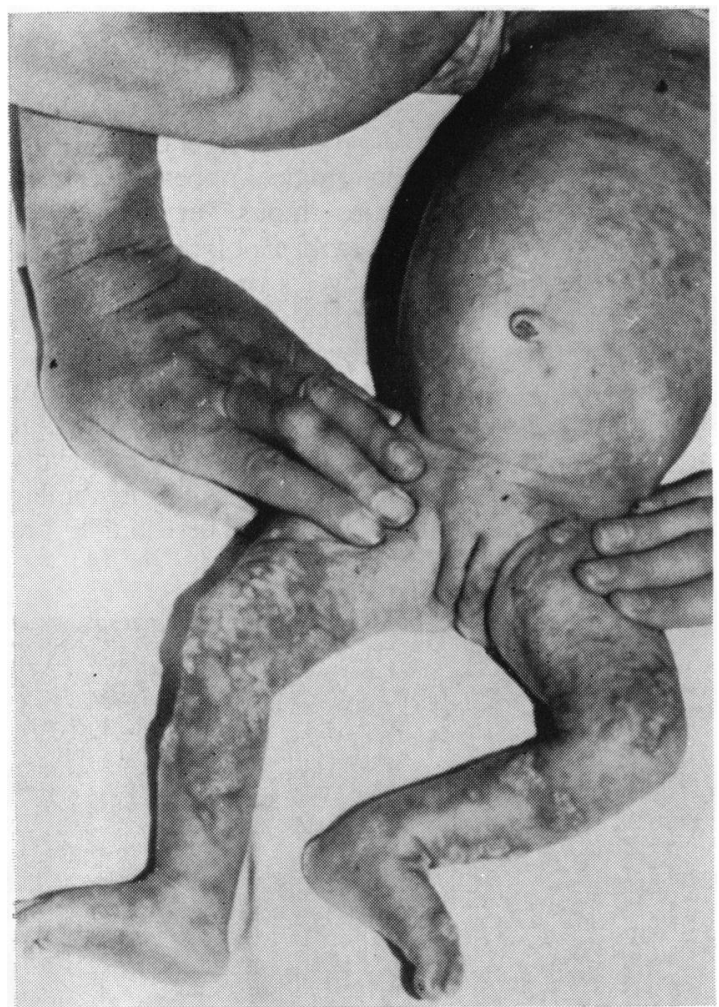

Fig. 1 Skin lesions at 3 months of age. Bullous eruptions on lower limbs and chest which have started becoming pigmented.

anterior segments were normal. Her visual acuity was $6 / 5$ in the left eye and 6/18 in the right eye, improving to $6 / 9$ with $-0.75 \mathrm{D}$ sphere. The left fundus was normal. The right fundus was seen through clear media. There were a few microaneurysms temporal to the macula. A venule just temporal to the macula and arising from the inferior temporal vein was slightly elevated from the retinal plane. There was some preretinal fibrosis in this area (Fig. 3). In the upper temporal equatorial region there was extensive preretinal fibrosis and retinitis proliferans (Fig. 4). The other areas of the fundus showed no gross abnormality. Her visual field was full in the left eye, but the right peripheral field showed lower nasal loss consistent with the changes in the temporal fundus.

Fluorescein angiography of the right eye was done. It confirmed microaneurysms in the macular area connected to the raised venule (Fig. 5). Fluorescence persisted in the microaneurysms for more than 5 minutes, but there was no leakage from these or from the venule. The temporal retinitis proliferans rapidly filled with fluorescein (Fig. 6) and gave rise to profuse leakage of dye, which persisted for more than 10 minutes.

\section{Discussion}

More than 200 cases of IP have been reported since its first description by Bloch in 1926. Most of the children are full-term normal deliveries. There is no definite hereditary pattern, though some hereditary cases have been reported-for example, it has been described in a mother and her 2 daughters (Jackson and Nigam, 1962). Sulzberger (1938) described a case whose mother, sister, and niece suffered in

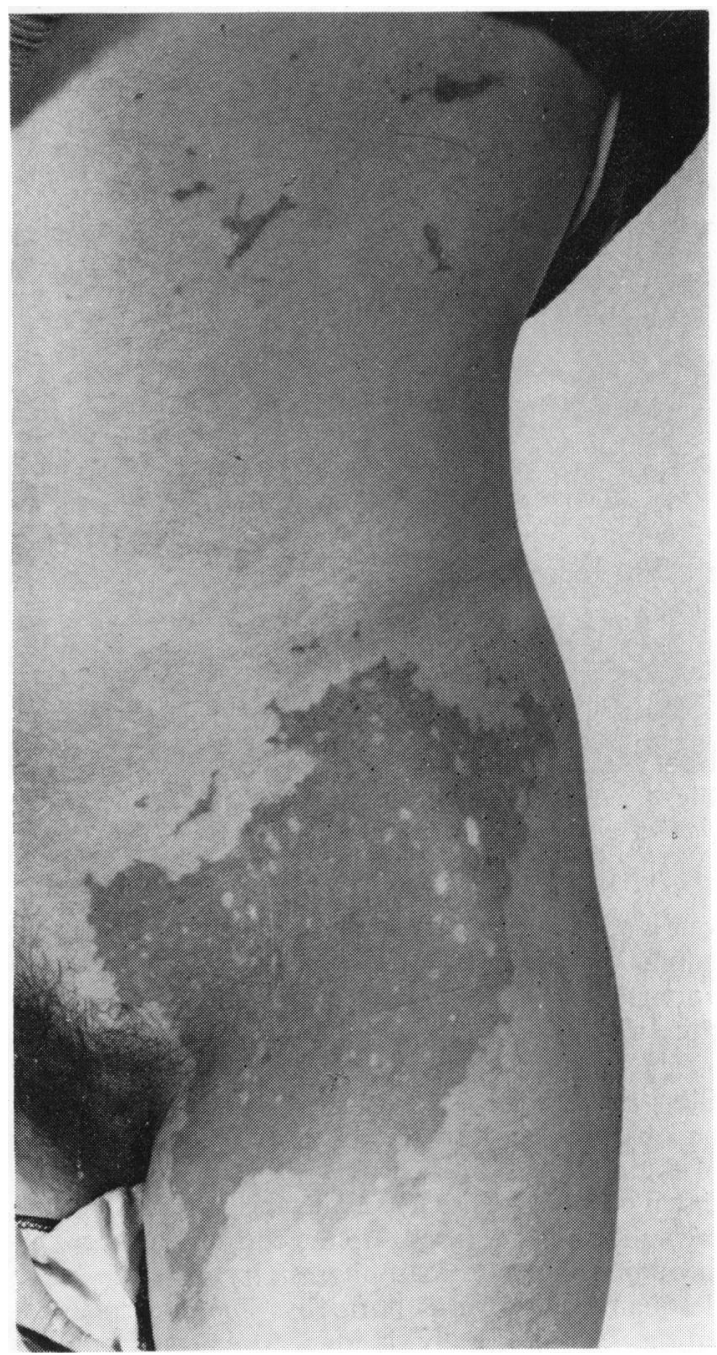

Fig. 2 Skin lesions at 15 years of age. Bizarre whorls and streaks of pigmentation on the trunk and the left groin 


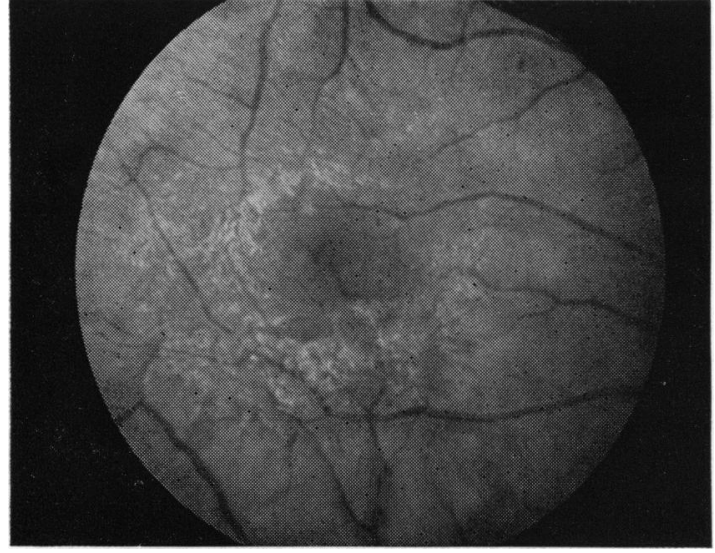

Fig. 3 Right macular area showing preretinal fibrosis. $A$ venule just temporal to the macula and arising from the inferior temporal vein is elevated from the retinal plane

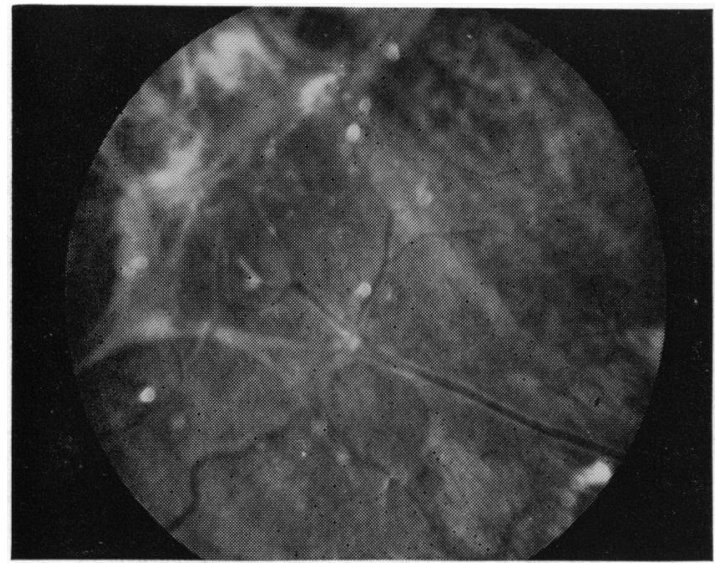

Fig. 4 The upper temporal equatorial area of right fundus showing preretinal fibrosis and retinitis proliferans

varying degrees from some congenital ectodermal anomalies. There is no family history of ectodermal anomaly in our case. There is a striking sex predilection, with girls being affected at a ratio of 20 to 1 .

The term incontinentia pigmenti was first used by Bloch in 1926 because on histological examination he observed abnormalities of the pigmented cells of the epithelium which were thought to be 'incontinent' of melanin. The pigment is not retained by melanoblasts but drops into the corium and accumulates in the chromatophores instead of being desquamated upwards normally. The hyperpigmentation seen clinically is due to chromatophores heavily laden with melanin and situated within the upper and middle cutis. Electron microscopic examination of pigment in one case (Mensheha-
Manhert et al., 1975) showed mostly mature and some immature melanin granules and more lipofuscin than would be expected normally. The microscopic picture is not diagnostic of the condition (Haber, 1952). Similar histological appearances also occur in lichen planus, lupus erythematosis, Civatte's poikiloderma, Jacobi's poikiloderma, and Riehl's melanosis. However, the peculiar and characteristic clinical pattern of pigmentation is typical of the Bloch-Sulzberger syndrome.

The primary skin condition is present at birth or develops soon afterwards. It appears to be a macular or diffuse erythema which is characteristically associated with lichenoid papules and bullae arranged irregularly or in stripes. These bullae are

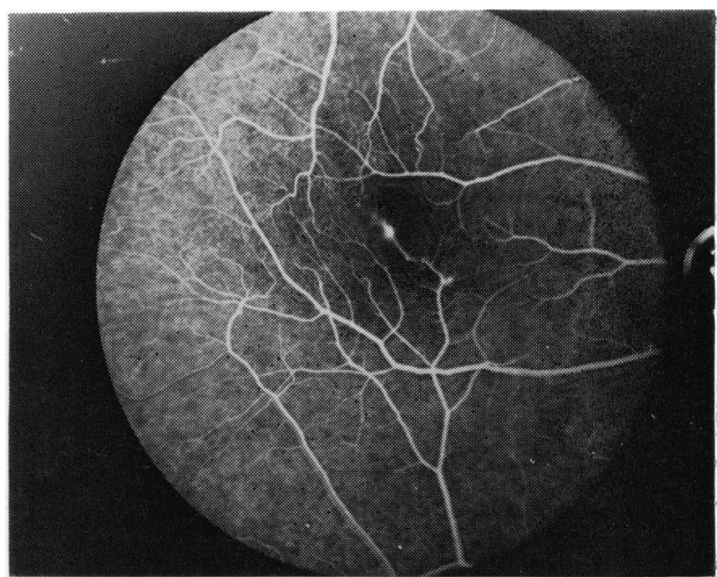

Fig. 5 Fluorescein angiogram showing microaneurysms connected to the raised venule temporal to the right macula

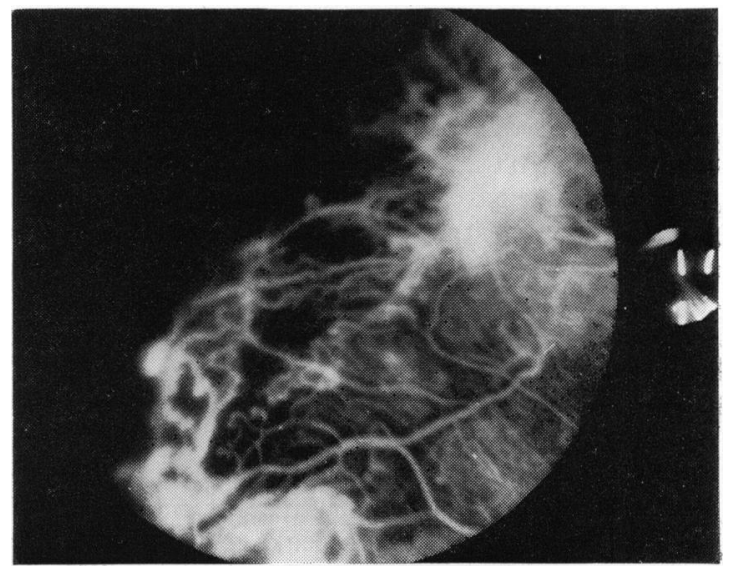

Fig. 6 Retinitis proliferans in temporal equatorial region rapidly fills up with fuorescein 
liable to rupture and may lead to oozing and crusting. This stage is soon replaced by typical skin pigmentation. The pigmentary stage persists for a number of years, after which the pigment tends to disappear spontaneously.

Abnormalities of hairs and dentition are associated features of IP and they were both present in our case. In Bloch-Sulzberger syndrome there may be alopecia at the vertex with atrophy of skin in this area. The hairs in such areas are short and coarse. Delayed appearance of teeth of poor quality is often found in these patients. Variation in shape may also occur, as conical or irregular configurations. Total failure of tooth bud development has also been described.

Eye abnormalities have been noted in one-quarter to one-third of patients with this disease. Both anterior and posterior segment changes may occur. The anterior segment changes include cataract, uveitis, and blue sclerae. Pigmentation of the bulbar conjunctiva in the region of interpalpebral aperture has been described (McCrary and Smith, 1968), which histologically resembled the skin pigments. Nystagmus, strabismus, myopia, microphthalmos, and phthisis bulbi have also been described. A number of cases of IP have been reported with associated posterior segment changes which include optic atrophy (Carney, 1951; Lapiere, 1951), papillitis (Kuhling, 1949), persistent hyperplastic primary vitreous, metastatic ophthalmitis (Kawamura, 1954; Scott and others, 1955). ablatio falciformis, retrolental fibroplasia with or without pseudoglioma, retinal dysplasia (Findlay, 1952; Cole and Cole, 1959; Jones, 1966; MenshehaManhert and others, 1975; Miller and Anderson, 1966; Zweifach, 1966). About one-third of the ocular defects involve a mass in the posterior chamber. The retinal dysplasia and associated retinal detachment seen in IP may be secondary to the underlying abnormalities of retinal pigment epithelium, which in turn may reasonably be considered an expression of the underlying disorder of pigmentation.

Abnormal retinal pigmentation was described by Fischbein et al. (1972) in the form of small patches of depigmentation and pigment variegation scattered diffusely throughout the fundi. McCrary and Smith (1968) found patchy mottling of pigment in the midzone of the fundus in both eyes of a patient. This pigment proliferation and loss appear similar to the pattern of skin changes. Both these authors also performed fluorescein angiography in their patients, which showed bright fluorescence in the depigmented patches due to choroidal flush.

Findlay (1952) described a case with oedema of the posterior pole. All the retinal blood vessels and the whole choroidal bed were grossly dilated with several areas of punctate haemorrhages. Jensen
(1956) reported a girl with a large oblique whitish membrane-like formation proliferating into the vitreous with vascularised processes to the retina. Its posterior portion concealed the optic disc. The adjacent retinal zone was slightly depigmented, while the peripheral retina showed diffuse dense pigmentation. In addition there were 2 small, welldefined retinal haemorrhages.

Lieb and Guerry (1958) described a case of exudative chorioretinitis associated with IP in a 4-week-old girl. The right fundus showed engorgement of the superior and inferior temporal veins. Temporal to the macula one branch of inferior temporal vein was markedly tortuous and engorged, resembling a corkscrew in configuration. The superior temporal vein showed multiple divisions resembling a pannus-like vascularisation. The whole temporal retina was oedematous, especially in the area of neovascularisation. In the left fundus the macular area showed extensive oedema and corkscrew vessels.

The fundus changes observed in our case have not previously been described in IP. Microaneurysms in the macular area were not reported in the earlier cases. The case reported by Lieb and Guerry (1958), like our case, also showed an abnormal branch of the inferior temporal vein temporal to the macula. The venule was elevated with some preretinal fibrosis, which formed a circinate pattern around the macular area. It did not show any leakage on fluorescein angiography. There was slight pigmentary disturbance in the macular area but no retinal oedema and no retinal haemorrhages. There were fine exudates in a fan shape between the macula and the retinitis proliferans temporally.

Although a number of cases of retinal dysplasia and retinal detachment have been reported, only 1 case (Jensen, 1956) showed connective tissue proliferation projecting into the vitreous. The patient described here has definite retinitis proliferans circumferentially in the equatorial region. There was sheathing along the superior temporal vessels and their branches, which on the temporal side became continuous with the retinitis proliferans. There was abnormal branching of the superior temporal vessels in the region of the retinitis proliferans. The retinitis proliferans showed marked leakage of fluorescein. The retinal changes were limited to the right eye. The fundus picture has not changed much in the past $1 \frac{1}{2}$ years under observation.

\section{References}

Bloch, B. (1926). Eigentümliche bisher nicht beschreibene Pigmentaffektion (incontinentia pigmenti). Schweizerischemedizinische Wochenschrift, 7, 404-405.

Carney, R. G. (1951). Incontinentia pigmenti-a report of 
five cases and review of literature. Archives of Dermatology and Syphilology, 64, 126-135.

Cole, J. G., and Cole, H. G. (1959). Incontinentia pigmentiassociated with changes in the posterior chamber of the eye. American Journal of Ophthalmology, 47, 321-331.

Findlay, G. H. (1952). On the pathogenesis of incontinentia pigmenti-with observation on an associated eye disturbance resembling retrolental fibroplasia. British Journal of Dermatology, 64, 141-146.

Fischbein, F. I., Schub, M., and Lesko, W. S. (1972). Incontinentia pigmenti, pheochromocytoma and ocular abnormalities. American Journal of Ophthalmology, 73, 961-964.

Haber, H. (1952). The Bloch-Sulzberger syndrome (incontinentia pigmenti). British Journal of Dermatology, 64, $129-140$.

Jackson, R., and Nigam, S. (1962). Incontinentia pigmentia report of three cases in one family. Pediatrics, 30, 433442 .

Jensen, V. A. (1956). Incontinentia pigmenti (Bloch-Sulzberger syndrome) associated with proliferative eyeground changes and positive toxoplasmosis reaction. Acta Psychiatrica Scandinavica, 31, 197-202.

Jones, S. T. (1966). Retrolental membrane associated with Bloch-Sulzberger syndrome (incontinentia pigmenti). American Journal of Ophthalmology, 62, 330-334.

Kawamura (1954). Cited by Miller, R. J., and Anderson, R. E. Incontinentia pigmenti in Japan. Archives of Dermatology and Syphilology, 69, 667.
Kuhling, F. (1949). Cited by Lieb, W. A., and Guerry, D. Incontinentia Pigmenti. Dissertation, Wurzburg.

Lapiere, S. (1951). Cited by Lieb, W. A., and Guerry, D. Incontinentia Pigmenti. Archives Belges de Dermatologie et de Syphiligraphy, 7, 156.

Lieb, W. A., and Guerry, D. (1958). Fundus changes in incontinentia pigmenti. American Journal of Oplthalmology, 45, 265-271.

McCrary, J. A., and Smith, J. L. (1968). Conjunctival and retinal incontinentia pigmenti. Archives of Ophthalmology, 79, 417-422.

Mensheha-Manhert, O., Rodrignes, M. M., Shields, J. A., Shannon, G. M., and Mirabelli, R. P. (1975). Retinal pigment epithelium in incontinentia pigmenti. American Journal of Ophthalmology, 79, 571-577.

Miller, R. J., and Anderson, R. E. (1966). A retrolental mass in incontinentia pigmenti-case report and review of literature. Survey of Ophthalmology, 11, 41-46.

Scott, J. G., Friedmann, A. I., Chitters, M., and Pepler, W. I. (1955). Ocular changes in the Bloch-Sulzberger syndrome (incontinentia pigmenti). British Journal of Ophthalmology, 39, 276-282.

Sulzberger, M. B., Frazer, J. F., and Hunter, L. (1938). Incontinentia pigmenti. Archives of Dermatology and Syphilology, 38, 57.

Zweifach, P. H. (1966). Incontinentia pigmenti: its association with retinal dysplasia. American Journal of Ophthalmology, 62, 716-722. 